\title{
SEROPREVALENCIA POBLACIONAL DE LA ENFERMEDAD CELIACA EN ZONAS URBANAS DEL PERÚ
}

\author{
Katherine Baldera $\mathbb{1}^{1, a}$, David Chaupis-Meza $\mathbb{1}^{1, b}$, César Cárcamo $\mathbb{1}^{2, \mathrm{c}}$, \\ King Holmes (10), , Patricia García $\mathbb{1}^{2, e}$ \\ 1 Escuela de Tecnología Médica de la Facultad de Medicina, Universidad Peruana Cayetano Heredia, Lima, Perú. \\ 2 Unidad de Epidemiología, ETS y VIH de la Facultad de Salud Pública y Administración, Universidad Peruana \\ Cayetano Heredia, Lima, Perú. \\ ${ }^{3}$ School of Public Health and Community Medicine, University of Washington, Seattle, Washington, EE. UU. \\ a Licenciada en Tecnología Médica, especialidad de Laboratorio Clínico; ${ }^{\mathrm{b}}$ bachiller en Tecnología Médica; ${ }^{\mathrm{c}}$ médico, \\ doctor en Epidemiología; ${ }^{\mathrm{d}}$ médico, doctor en Microbiología; ${ }^{\mathrm{e}}$ médico, doctora en Medicina.
}

\section{RESUMEN}

El objetivo del estudio fue determinar la seroprevalencia de la enfermedad celiaca (EC) en zonas urbanas del Perú, utilizando una muestra de base poblacional. Se tamizó una muestra aleatoria de mujeres y varones de 18 a 29 años de 26 ciudades del Perú. Para la detección de la EC se utilizó el kit anti-transglutaminasa tisular IgA. Los resultados mayores a $20 \mathrm{AU} / \mathrm{ml}$ fueron considerados positivos. La prevalencia ponderada de la EC fue de 1,2\% (IC 95\%: 0,0-2,4) y se estima que el número de personas viviendo con EC en el Perú fue de 341 783. La prevalencia de la EC en el Perú resultó ser similar al promedio mundial.

Palabras clave: Enfermedad Celiaca; Seroprevalencia; Estudios Seroepidemiológicos; Transglutaminasas (fuente: DeCs BIREME).

\section{POPULATION SEROPREVALENCE OF CELIAC DISEASE IN URBAN AREAS OF PERU}

\begin{abstract}
The objective of the study was to determine the seroprevalence of celiac disease (CD) in urban areas of Peru using a population-based sample. A random sample of women and men 18 to 29 years old from 26 cities in Peru was screened. An anti-tissue transglutaminase IgA kit was used for the detection of CD. Results higher than $20 \mathrm{AU} / \mathrm{ml}$ were considered positive. The weighted prevalence of celiac disease was $1.2 \%$ (CI 95\%: $0.0 \%$ - 2.4\%), thus the estimated number of people living with CD in Peru was 341,783. CD prevalence in Peru is similar to the world average.
\end{abstract}

Keywords: Celiac Disease; Seroprevalence; Seroepidemiologic Studies; Transglutaminases (source: MeSH NLM).

\section{INTRODUCCIÓN}

Citar como: Baldera K, ChaupisMeza D, Cárcamo C, Holmes K, García P. Seroprevalencia poblacional de la enfermedad celíaca en zonas urbanas del Perú. Rev Peru Med Exp Salud Publica. 2020;37(1):636. Doi: https://doi.org/10.17843/ rpmesp.2020.371.4507

Correspondencia: David Chaupis Meza; Universidad Peruana Cayetano Heredia; Av. Honorio Delgado 430, Lima 31, Perú; david.chaupis.m@upch.pe

Recibido: 02/05/2019

Aprobado: 29/01/2020

En línea: 23/03/2020
La intolerancia al gluten (sea por trigo, cebada o centeno) conlleva a un amplio espectro de enteropatías crónicas: a) alergia al gluten, b) sensibilidad no celiaca al gluten (SNCG) y c) enfermedad celiaca (EC). La EC es un trastorno autoinmune con predisposición genética vinculada al alelo HLA, DQ2 y DQ8 ${ }^{(1,2)}$. Pese a su reconocida caracterización, su diagnóstico siempre es esquivo.

La SNCG no se asocia a ninguna alteración genética ni inmunológica como la EC, sin embargo, ambas condiciones presentan un cuadro clínico similar. Aparentemente esto responde a que un fragmento derivado del gluten, la alfa-gliadina, sería el principal blanco epitópico asociado a los trastornos duodenales presentes tanto en la EC como la SNCG ${ }^{(3)}$.

Según su presentación clínica, la EC puede presentarse con evidente sintomatología (diarrea, esteatorrea, fatiga, dispepsia y desnutrición); un cuadro clínico extragastrointestinal (anemia ferropénica, fatiga, depresión, neuropatías periféricas y ataxia cerebelosa); o un cuadro asintomático ${ }^{(4)}$, detectado muchas veces por un tamizaje serológico rutinario o por su asociación a complicaciones crónicas como linfomas malignos ${ }^{(5)}$.

La prevalencia de la EC a nivel mundial es de 1\%, con una razón mujer:hombre de 2,8:1. Se presume que en Latinoamérica las prevalencias son similares a las de Europa, las cuales 
varían entre 0,46 y $0,64 \%{ }^{(6)}$. En el Perú no existen estudios poblacionales de prevalencia, pero sí hay casos clínicos de EC que advierten la necesidad de un mapeo serológico nacional de la enfermedad ${ }^{(7,8,9)}$.

Estas pruebas detectan anticuerpos reactivos al gluten (IgA y/o IgG), tales como: anti endomisio (EMA), antigliadina (AGA) y antitransglutaminasa (tTGA). Este último es usado para la detección temprana de la EC a cualquier edad, confirmándose luego por una biopsia intestinal ${ }^{(10)}$. La finalidad del presente estudio fue determinar la seroprevalencia de la EC en poblaciones urbanas del Perú utilizando una muestra de base poblacional.

\section{EL ESTUDIO}

\section{Sujetos y población de estudio}

El presente estudio utiliza información demográfica y muestras biológicas del estudio PREVEN ${ }^{(11)}$, que incluyó mujeres y varones entre 18 y 29 años de 26 ciudades del Perú, seleccionados a través de un muestreo por conglomerados multietápico en viviendas de zonas urbanas. Nuestro estudio seleccionó 1208 muestras por muestreo aleatorio simple de 17293 recolectadas entre el 2005 y 2007 por el estudio PREVEN. Los participantes completaron un cuestionario epidemiológico y proporcionaron muestras biológicas. El suero obtenido se mantiene almacenado a $-20{ }^{\circ} \mathrm{C}$ en el banco de sueros del Laboratorio de Salud Global de los Laboratorios de Investigación y Desarrollo (LID) de la Universidad Peruana Cayetano Heredia (UPCH).

\section{Procesamiento de muestras}

Se descartaron muestras hemolizadas, lipémicas; así como también crioviales con un volumen remanente insuficiente $(<10 u l)$ de suero o muestras sin información epidemiológica. Las muestras fueron procesadas mediante la metodología inmunoenzimática colorimétrica (ELISA). Se usó el kit antitransglutaminasa tisular IgA (Diametra Diagnostic, Italia) de acuerdo con el protocolo del fabricante, cuya sensibilidad es de $93 \%$ y especificidad de $95 \%{ }^{(12)}$. Las muestras y el kit de reactivos se descongelaron a temperatura ambiente alrededor de 30 minutos. Se incluyó un control positivo y un control negativo. Las muestras fueron diluidas 1:10 y colocadas en los pocillos recubiertos con transglutaminasa tisular recombinante. Se incubaron durante 30 minutos a temperatura ambiente.

Se descartó el sobrenadante y se lavó tres veces los micropocillos para eliminar el resto de los componentes no unidos. Después se agregó el conjugado de anti-IgA humano marcada con peroxidasa de rábano picante (HRP) y se incubó por 30 minutos a temperatura ambiente. Tras un lavado se eliminó el conjugado sobrante, se agregó el substrato tetrametilbencidina (TMB) y se incubó 15 minutos a tempe-

\section{MENSAJES CLAVE}

Motivación para realizar el estudio: La celiaquía es una enfermedad con diferentes presentaciones clínicas, siendo los casos no diagnosticados la base del iceberg por reconocer. Un tamizaje serológico puede ser una prueba de detección temprana para la celiaquía; además, evitaría complicaciones mortales.

Principales hallazgos: Este es el primer estudio en Perú de base poblacional «extranosocomial». Se encontró una prevalencia de enfermedad celiaca del 1,2\% usando la prueba de anti-transglutaminasa tisular IgA.

Implicancias: Se recomienda realizar más estudios a nivel poblacional de la enfermedad celiaca en Perú.

ratura ambiente al resguardo de la luz. La reacción se detuvo con ácido sulfúrico.

La lectura de la muestra se hizo con un filtro $450 \mathrm{~nm}$ y un filtro de referencia de $650 \mathrm{~nm}$. Como prueba confirmatoria, aquellas muestras con resultados $>20 \mathrm{AU} / \mathrm{ml}$ fueron sometidas a un segundo ELISA siguiendo los mismos procedimientos descritos. Aquellas muestras en las que el segundo ELISA mostró valores $>20 \mathrm{AU} / \mathrm{ml}$ fueron consideradas positivas. Las muestras con valores $\leq 20 \mathrm{AU} / \mathrm{ml}$ en el primer o segundo ELISA fueron consideradas negativas.

\section{Análisis estadístico}

Para cada muestra incluida en el estudio se calculó un factor de expansión, correspondiente a la inversa de la probabilidad de participación. La probabilidad de participación fue el producto de varias probabilidades: la probabilidad de selección del conglomerado dentro de la ciudad, la probabilidad de selección de la vivienda dentro del conglomerado, la probabilidad de selección del participante dentro de la vivienda, y la probabilidad de selección del individuo para su participación en el presente estudio. Los estimados también fueron ajustados por muestreo estratificado a nivel ciudad. Se presentan prevalencias ponderadas, con sus respectivos intervalos de confianza. Para la comparación de proporciones se usó la prueba de chi cuadrado de Pearson para muestras ponderadas. Para todos los cálculos se usó el programa estadístico Stata, versión 8.2 (College Station, Texas).

\section{Aspectos éticos}

Los participantes proporcionaron consentimiento verbal para su participación en el estudio. El formato de consentimiento informado del estudio original contemplaba el almacenamiento de las muestras para estudios futuros sin la inclusión de identificadores personales. Como parte de este estudio solo se utilizaron códigos para identificar las muestras y ligarlas a su información epidemiológica. El presente estudio tuvo la aprobación del Comité Institucional de Ética 
en Investigación de la Universidad Peruana Cayetano Heredia (código SIDISI 60226).

\section{HALLAZGOS}

De 1208 muestras seleccionadas, se descartaron 107 por estar hemolizadas (73), lipémicas (1), volumen insuficiente (18) y ausencia de información epidemiológica (15). Finalmente se incluyeron 1101 muestras en el análisis, de estas 420 fueron de varones y 681 de mujeres (Tabla 1).

Se obtuvo una prevalencia ponderada de EC de 1,2\% (IC 95\%: 0,0-2,4). La muestra es representativa de 3399734 personas de 18 a 29 años, residentes de áreas urbanas en Perú. De tal manera que se estima que en esa población existen 40797 personas con EC (IC 95\%: 0-81 594).

Si bien la prevalencia ponderada en mujeres $(0,7 \%)$ fue menor que en varones (1,9\%), esta diferencia no es estadísticamente significativa $(\mathrm{p}=0,253)$. Del mismo modo, la prevalencia en la categoría de edad de 21 a 23 años es mayor que para otros grupos etarios, no se encuentra diferencia estadísticamente significativa $(\mathrm{p}=0,144)$.

Del total de individuos, 623 pertenecían a la región de la costa, 281 a la sierra y 197 a la selva. La prevalencia de EC en la sierra $(1,8 \%)$ no fue significativamente mayor a la encontrada en la costa o en la selva ( 1,1 y $0,9 \%$, respectivamente).

Si bien, pocos participantes hablaban quechua o aymara (119 y 19, respectivamente), no se encontró diferencias estadísticamente significativas en la prevalencia de la EC entre

Tabla 1. Características demográficas de los 1101 participantes seleccionados para el estudio

\begin{tabular}{lcc}
\hline \multirow{2}{*}{ Variable } & Tamizados & Prevalencia $^{\mathrm{a}}$ \\
\cline { 2 - 3 } Sexo & $\mathbf{n}=\mathbf{1 1 0 1}$ & $\mathbf{n}(\%)$ \\
Masculino & 420 & $6(1,9)$ \\
Femenino & 681 & $9(0,7)$ \\
Edad (años) & & \\
18-20 & 353 & $8(0,9)$ \\
$21-23$ & 261 & $3(2,6)$ \\
$24-26$ & 233 & $1^{\mathrm{b}}$ \\
$27-29$ & 254 & $3(0,7)$ \\
Región & & \\
Costa & 623 & $7(1,1)$ \\
Sierra & 281 & $5(1,8)$ \\
Selva & 197 & $3(0,9)$ \\
Lengua nativa & & $3^{\mathrm{b}}$ \\
Quechua & 119 & $0^{\mathrm{b}}$ \\
Aymara & 19 &
\end{tabular}

${ }^{\text {a }}$ Prevalencia ponderada; ${ }^{\mathrm{b}}$ no se pudo calcular al haber solo una unidad primaria de muestreo estos y el resto de los participantes en el estudio $(\mathrm{p}=0,288 \mathrm{y}$ $\mathrm{p}=0,811$, respectivamente).

\section{DISCUSIÓN}

Este es el primer estudio en Latinoamérica que evalúa la prevalencia de la EC mediante tamizaje serológico para IgA anti-transglutaminasa tisular en una muestra con base poblacional, encontrando una prevalencia de $1,2 \%$. Si la prevalencia para otras edades y áreas geográficas del país en el 2007 hubiera sido la misma, entonces se estima que para ese año el número de personas viviendo con enfermedad celiaca en el Perú fue de 341783.

En los Estados Unidos, la prevalencia de EC no diagnosticada estaría en incremento. En este país, un estudio determinó la presencia de anti-transglutaminasa tisular IgA en muestras almacenadas (por al menos 20 años a $-20^{\circ} \mathrm{C}$ ), para determinar cambios en la prevalencia en un periodo de 50 años ${ }^{(13)}$. Muestras extraídas entre 1948 a 1954 evidencian una prevalencia del 0,2\% y aquellas extraídas entre 1995 a 2003 señalan una prevalencia de $0,8 \%$, ambas en adultos mayores de 50 años. Por otro lado, en muestras de personas con edades entre 18 a 49 años tomadas entre 2006 a 2008 se encontró una prevalencia de $0,9 \%$. Previamente en el estudio se reveló la presencia de IgA en las muestras antiguas usando nefelometría.

Otro estudio realizado en Argentina ${ }^{(14)}$, utilizando AGA (IgA e IgG) y EmA (IgA) como marcadores serológicos, determinó una prevalencia de $0,6 \%$ de EC. Nuestro estudio revela una mayor prevalencia, probablemente por el grupo etario y el tipo de prueba serológica utilizada. Por otra parte, un estudio en Brasil ${ }^{(15)}$ en personas de 18 a 65 años sin anemia encontró una prevalencia de $0,33 \%$ usando la prueba de anti-transglutaminasa tisular IgA y anti-EmA IgA. La baja prevalencia encontrada podría responder al hecho de que la anemia es una presentación atípica de la EC.

Los estudios epidemiológicos que exploran el riesgo poblacional de presentar la EC son escasos en Latinoamérica ${ }^{(6)}$. En el Perú, los pocos reportes clínicos que existen advierten la presencia de la EC en una «baja frecuencia» y ligada al tipo clásico de la enfermedad ${ }^{(7-9,16)}$.

El tamizaje serológico para la EC está recomendado en vez del uso de métodos más invasivos como las biopsias, siendo una elección de diagnóstico temprano para descartar la EC. Inclusive, según la ESPGHAN (European Society for Paediatric Gastroenterology Hepatology and Nutrition) ${ }^{(17)}$ se puede confirmar el diagnóstico de la EC con niveles de antitransglutaminasa por encima de diez veces a los límites superiores normales ( $\geq 20 \mathrm{U} / \mathrm{ml}$, valor referencial para nuestro estudio), cuyos niveles se comparan a una atrofia vellositaria tipo Marsh-3. Esto enfatiza la utilidad de la prueba serológica tTGA IgA como marcador serológico más confiable para la EC, no solo por su alta especificidad y sensibilidad, sino por su utilidad con muestras almacenadas por largos periodos, 
siendo posible su reutilización en muestras criopreservadas ${ }^{(18)}$, como en nuestro caso. En efecto, contar con una prueba serológica confiable permitiría reducir costos y tiempo en el diagnóstico de la EC.

La ausencia de información sobre síntomas gastrointestinales e historia de EC podría constituir una limitación del presente estudio, sin embargo, la correlación clínica de la serología es bastante conocida.

Nuestro estudio encontró una mayor prevalencia de EC en habitantes de la sierra. Esto podría corresponder a diferencias genéticas, o a factores ambientales, tales como la altura y la consecuente hipoxia. Si bien otros estudios han encontrado prevalencias mayores en mujeres ${ }^{(6)}$, en nuestro estudio el sexo más afectado es el masculino, diferencia para la cual no encontramos causa.

En conclusión, el presente estudio es el primero realizado en el Perú con base en una muestra poblacional en adultos jóvenes, evidenciándose una prevalencia mayor a la reportada en otros estudios del continente americano pero similar al promedio mundial. Se sugiere más estudios de seroprevalencia en poblaciones atípicas a la EC, teniendo en cuenta sus distintas formas de presentación clínica, debido a que en la mayoría de los casos la celiaquía no evidencia sintomatología aparente.

Contribución de autoría: KB participó en la ejecución técnica, análisis de datos y obtuvo el financiamiento del estudio. DCM participó en la concepción, diseño del estudio, interpretación de los resultados y redacción de la primera versión del manuscrito. CC participó en el análisis e interpretación estadística, revisión crítica y redacción de la versión final del artículo. Tanto KH y PG son los investigadores principales del estudio PREVEN. Todos los autores aprobaron finalmente la última versión del artículo.

Financiamiento: Los autores declaran que el estudio fue financiado por la Fundación Instituto Hipólito Unanue.

Conflictos de interés: Los autores declaran que no tienen conflictos de interés en la publicación del artículo.

\section{REFERENCIAS BIBLIOGRÁFICAS}

1. Sapone A, Bai JC, Ciacci C, Dolinsek J, Green PHR, Hadjivassiliou M, et al. Spectrum of gluten-related disorders: consensus on new nomenclature and classification. BMC Med. 2012;10:13.

2. Elli L, Branchi F, Tomba C, Villalta D, Norsa L, Ferretti F, et al. Diagnosis of gluten related disorders: Celiac disease, wheat allergy and non-celiac gluten sensitivity. World J Gastroenterol. 2015;21(23):7110-9.

3. Lebwohl B, Ludvigsson JF, Green PHR. Celiac disease and non-celiac gluten sensitivity. BMJ. 2015;351:h4347.

4. Rashid M, Lee J. Serologic testing in celiac disease: Practical guide for clinicians. Can Fam Physician Med Fam Can. 2016;62(1):38-43.

5. Kelly CP, Bai JC, Liu E, Leffler DA. Advances in diagnosis and management of celiac disease. Gastroenterology. 2015;148(6):1175-86.

6. Parra-Medina R, Molano-Gonzalez N, Rojas-Villarraga A, Agmon-Levin N, Arango M-T, Shoenfeld Y, et al. Prevalence of celiac disease in latin america: a systematic review and meta-regression. PloS One. 2015;10(5):e0124040.

7. Vera A, Frisancho O, Yábar A, Carrasco W. Enfermedad Celiaca y Obstrucción Intestinal por Linfoma de Células T. Rev Gastroenterol Peru. 2011;31(3):278-81.

8. Llanos O, Matzumura M, Tagle M, Huerta-Mercado J, Cedrón H, Scavino J, et al. Enfermedad celiaca: estudio descriptivo en la Clínica Anglo Americana. Rev Gastroenterol Peru. 2012;32(2):134-40.

9. Tagle M, Nolte C, Luna E, Scavino Y. Coexistencia de Enfermedad Celíaca y Hepatitis Autoinmune. Reporte de un caso y revisión de la literature. Rev Gastroenterol Peru. 2006;26(1):80-3.

10. Rostom A, Murray JA, Kagnoff MF. Medical Position Statement on Celiac Disease. Gastroenterology. 2006;131(6):1977-80.
11. García PJ, Holmes KK, Cárcamo CP, Garnett GP, Hughes JP, Campos PE, et al. Prevention of sexually transmitted infections in urban communities (Peru PREVEN): a multicomponent community-randomised controlled trial. Lancet. 2012;379(9821):1120-8.

12. Kowalski K, Mulak A, Jasinska M, Paradowski Leszek. Diagnostic challenges in celiac disease. Adv Clin Exp Med. 2017; 26(4): 729-37.

13. Rubio-Tapia A, Ludvigsson JF, Choung RS, Brantner TL, Rajkumar SV, Landgren $\mathrm{O}$, et al. Increased mortality among men aged 50 years old or above with elevated IgA anti-transglutaminase antibodies: NHANES III. BMC Gastroenterol. 2016;16(1):136.

14. Gomez JC, Selvaggio GS, Viola M, Pizarro B, la Motta G, de Barrio S, et al. Prevalence of celiac disease in Argentina: screening of an adult population in the La Plata area. Am J Gastroenterol. 2001;96(9):2700-4.

15. Melo SBC, Fernandes MIM, Peres LC, Troncon LEA, Galvão LC. Prevalence and demographic characteristics of celiac disease among blood donors in Ribeirão Preto, State of São Paulo, Brazil. Dig Dis Sci. 2006;51(5):1020-5.

16. Arévalo F, Roe E, Arias-Stella-Castillo J, Cárdenas J, Montes P, Monge E. Low serological positivity in patients with histology compatible with celiac disease in Perú. Rev Esp Enferm Dig. 2010;102(6):372-5.

17. Smarrazzo A, Misak Z, Costa S, Mičetić-Turk D, Abu-Zekry M, Kansu A, et al. Diagnosis of celiac disease and applicability of ESPGHAN guidelines in Mediterranean countries: a real life prospective study. BMC Gastroenterol. 2017;17(1):17.

18. Wengrower D, Doron D, Goldin E, Granot E. Should stored Serum of Patients Previously Tested for Celiac Disease Serology be Retested for Transglutaminase Antibodies? J Clin Gastroenterol. 2006;40(9):806-8. 